\title{
Application of blood pre-albumin and NT-pro BNP levels in evaluating prognosis of elderly chronic heart failure patients
}

\author{
LINYING SHI, YUAN ZHANG, JING ZHANG，YUANFENG GAO，JIAMEI LIU, \\ MULEI CHEN and XINCHUN YANG
}

Department of Cardiology, Beijing Chaoyang Hospital, Beijing 100020, P.R. China

Received November 5, 2019; Accepted March 3, 2020

DOI: $10.3892 /$ etm.2020.8865

\begin{abstract}
Levels of blood pre-albumin (PA) and N-terminal pro-B-type natriuretic peptide (NT-pro BNP) in elderly patients with chronic heart failure (CHF) and their clinical value in prognosis evaluation were explored. A total of 410 elderly patients aged $\geq 65$ years hospitalized for CHF were enrolled. The concentrations of blood PA and NT-pro BNP, routine blood test and biochemistry indicators were determined and color Doppler echocardiography was performed. Additionally, the patients were followed up after discharge, and based on the occurrence of major adverse cardiac events (MACE), they were divided into MACE group and non-MACE group. MACE group had an older age and a higher level of plasma NT-pro BNP than non-MACE group $(77.82 \pm 6.57)$ years vs. $(76.39 \pm 6.18)$ years, and $(8,864.52 \pm 9,718.36) \mathrm{pg} / \mathrm{ml}$ vs. $(4,165.62+6,437.28) \mathrm{pg} / \mathrm{ml}(\mathrm{P}<0.05)$, and the left ventricular ejection fraction and serum PA level in MACE group were lower than those in non-MACE group $(\mathrm{P}<0.05)$. According to the results of multivariate regression analysis, serum PA [odds ratio $(\mathrm{OR})=0.242,95 \%$ confidence interval $(\mathrm{CI})=0.137-0.406, \mathrm{P}<0.001]$ and plasma NT-pro BNP $(\mathrm{OR}=1.847,95 \% \mathrm{CI}=1.024-3.158, \mathrm{P}=0.036)$ were independent risk factors for the occurrence of cardiac events during follow-up. Decline in PA level and elevation in NT-pro BNP level have a strong correlation with poor prognosis of elderly $\mathrm{CHF}$ patients, and they can be used for clinically evaluating disease conditions, guiding treatment and improving prognosis.
\end{abstract}

Correspondence to: Dr Mulei Chen or Dr Xinchun Yang, Department of Cardiology, Beijing Chaoyang Hospital, 8 Gongti South Road, Beijing 100020, P.R. China

E-mail: cml68@sina.cn

E-mail: yangxc@medmail.com.cn

Key words: pre-albumin, N-terminal pro-B-type natriuretic peptide, prognosis, the elderly, chronic heart failure, major adverse cardiac events

\section{Introduction}

Chronic heart failure (CHF) is a chronic disease, in which myocardial structure and function are changed due to cardiomyopathy, inflammation, myocardial infarction and other causes, ultimately leading to decline in ventricular pump function $(1,2)$. CHF occurs in the terminal stage of various heart diseases and is a major cause of death in heart disease patients, seriously endangering the life of patients. With the aging of populations and improvement of life expectancy in heart disease patients, CHF have become an important public health concern. Some studies have manifested that the incidence rate of $\mathrm{CHF}$ in the Chinese people aged $\geq 65$ years old is as high as $10 \%$, and elderly CHF patients tend to be complicated with malnutrition (3). With a half-life of about 2 days, pre-albumin (PA) is a sensitive indicator for monitoring nutritional status, and the decrease in PA level may be correlated with the poor prognosis of the patients with cardiovascular system disease, without related studies on its role in evaluating the prognosis of CHF (4-6). N-terminal pro-B-type natriuretic peptide (NT-pro $\mathrm{BNP}$ ) acts as a common indicator for the diagnosis of acute and $\mathrm{CHF}$ and the evaluation of their severity and has a significant correlation with left ventricular ejection fraction (LVEF), but it is more closely associated with age, volume load, renal function and diet habit $(7,8)$. The present study explored the significance of blood PA and NT-pro BNP for disease progression and prognosis in elderly $\mathrm{CHF}$ patients to accumulate data for clinical research of $\mathrm{CHF}$.

\section{Patients and methods}

Subjects. A total of 410 elderly patients aged $\geq 65$ years admitted to Beijing Chaoyang Hospital (Beijing, China) for CHF from January 2011 to June 2014 were enrolled in this study. There were 194 males and 216 females with the mean age of $(77.18 \pm 6.34)$ years. The patients were diagnosed with CHF based on the Framingham Criteria for Congestive Heart Failure, and their cardiac function was classified into grades II-IV (41 cases of grade II, 146 cases of grade III and 223 cases of grade IV) according to the New York Heart Association (NYHA) cardiac function classification standards. Exclusion criteria: i) Patients with malignant tumors, severe infection or tuberculosis, severe liver or kidney disease, thyroid disease, severe malnutrition or acute myocardial infarction, ii) those 
suffering from severe dehydration, gastrointestinal hemorrhage, shock or hypovolemia and iii) those who underwent major surgical operations in the last 3 months.

This study was approved by the Ethics Committee of Beijing Chaoyang Hospital. Patients who participated in this research had complete clinical data. The signed informed consents were obtained from the patients or the guardians.

General information. The general information of the subjects was collected, including the basic information of age, sex, routine blood test results, blood biochemical analysis results, echocardiograms and hospital stay. On the second day of admission, fasting venous blood was drawn to determine the serum PA and plasma NT-pro BNP concentrations and routine blood test and biochemistry indicators. The blood biochemistry indicators and PA were determined using the automatic biochemistry analyzer (Beckman Coulter, Inc.), and NT-pro BNP was measured using Elecsys 2010 fully automated electrochemiluminescence immunoassay analyzer (Roche Diagnostics). Echocardiography was performed in patients with Sonos 5500 color Doppler ultrasound system (Philips Medical Systems B.V.) within 3 days after admission. The probe frequency is $2-4 \mathrm{mhz}$. The machine is equipped with harmonic imaging technology (HI) and AQi technology analysis system. All the patients LVESD, LVEDD and other indicators were measured, and the LVEF indicator was calculated by Simpson's method.

Treatment and follow-up. All the patients received standardized treatment based on the '2016 ESC Guidelines for the Diagnosis and Treatment of Acute and Chronic Heart Failure'. After discharge, they were followed up by telephone or outpatient visit, and the follow-up lasted for 14-60 months with the occurrence of major adverse cardiac events (MACE) as the end of follow-up observation. The patients were divided into MACE group and non-MACE group according to the occurrence of MACE that is defined as re-hospitalization for heart failure and all-cause death.

Statistical analysis. All statistics were analyzed using SPSS 20.0 (IBM Corp.). The measurement data conforming to the normal distribution were expressed as (mean $\pm \mathrm{SD}$ ), and $\mathrm{t}$-test/z-test was performed for the comparisons between groups. The count data were presented as percentage or rate, and the comparisons between groups were made using Chi-square test. The variables with statistically significant differences were subjected to univariate logistic analysis, and multivariate logistic regression was used to analyze the potential risk factors. The receiver operating characteristics (ROC) curve analysis was used to analyze the diagnosis efficacy. $\mathrm{P}<0.05$ indicates statistical significance.

\section{Results}

Baseline characteristics. The comparisons indicated statistically significant differences in age, heart rate at admission, NYHA cardiac function classification, left ventricular diastolic dimension (LVEDD), LVEF, NT-pro BNP, albumin (ALB), PA, hemoglobin ( $\mathrm{Hb})$, hematocrit (Hct), total cholesterol (TC), triglyceride (TG), total bilirubin
(TBIL), serum creatinine (Scr) and cystatin C (Cys C) between the two groups $(\mathrm{P}<0.05$; Table I), and MACE group had an obviously higher level of plasma NT-pro BNP, but a notably lower level of serum PA than non-MACE group $[(8,864.52 \pm 9,718.36) \mathrm{pg} / \mathrm{ml}$ vs. $(4,165.62 \pm 6,437.28) \mathrm{pg} / \mathrm{ml}$, and $(0.14 \pm 0.07) \mathrm{g} / \mathrm{l}$ vs. $(0.19 \pm 0.05) \mathrm{g} / \mathrm{l} ; \mathrm{P}<0.001]$. However, there were no statistically significant differences in the sex, cardiac troponin I, D-dimer, alanine aminotransferase, aspartate aminotransferase, uric acid, hypersensitive C-reactive protein and hospital stay between the two groups $(\mathrm{P}>0.05$; Table I).

Results of logistic regression analysis. With the median as the boundary, the variables with statistically significant differences in baseline comparisons were classified into binary variables for univariate logistic regression analysis. According to the analysis results, NYHA cardiac function classification, LVEDD and NT-pro BNP had an OR value $>1$, and served as risk factors for the occurrence of MACE $(\mathrm{P}<0.05$; Table II), while with the OR values $<1$, LVEF, ALB, PA, Hb, Hct and TC were protective factors against MACE $(\mathrm{P}<0.05$; Table II). In the multivariate logistic regression analysis, despite of potential affecting factors indicated in Table III (NYHA, LVEDD, LVEF, ALB, Hb, Hct and TC), PA and NT-pro BNP were still statistically significant for the occurrence of MACE $(\mathrm{P}<0.05$; Table III), indicating that the decrease in PA and increase in NT-pro BNP have a strong correlation with chronic heart dysfunction to heighten the risk of such poor prognoses as all-cause death or re-hospitalization in elderly CHF patients.

Comparisons of heart failure severity and prognosis between $P A \leq 0.16$ g/l group and $P A>0.16$ g/l group. With the median of PA level as the boundary, the patients followed up were assigned into two groups, namely PA $\leq 0.16 \mathrm{~g} / 1$ group $(\mathrm{n}=198)$ and $\mathrm{PA}>0.16 \mathrm{~g} / \mathrm{l}$ group $(\mathrm{n}=212) . \mathrm{PA} \leq 0.16 \mathrm{~g} / \mathrm{l}$ group consisted of 98 males and 100 females, aged $(78.74 \pm 6.73)$ years on average, while PA $>0.16 \mathrm{~g} / 1$ group was composed of 102 males and 110 females, with the mean age of (76.21 \pm 6.16 ) years. PA $\leq 0.16 \mathrm{~g} / 1$ group had an older age and higher plasma NT-pro BNP level and incidence rate of MACE than PA $>0.16 \mathrm{~g} / 1$ group, and the differences were statistically significant $(\mathrm{P}<0.05$; Table IV), and the NYHA cardiac classification grade based on the percentages of cases of grades II-IV in PA $\leq 0.16 \mathrm{~g} / \mathrm{l}$ group were lower than that in PA $>0.16 \mathrm{~g} / \mathrm{l}$ group, with statistically significant differences $(\mathrm{P}<0.05$; Table IV).

Efficacy of serum NT-pro BNP and PA in the diagnosis of CHD patients. The sensitivity of NT-pro BNP in the diagnosis of CHF patients is $77.65 \%$, the specificity is $69.12 \%$, and the corresponding best cut-off point is $3,147.83 \mathrm{pg} /$ $\mathrm{ml}$; the sensitivity of PA in the diagnosis of CHF patients is $84.32 \%$, the specificity is $76.57 \%$, and the corresponding best cut-off point is $0.16 \mathrm{~g} / 1$; ROCAUCNT-pro BNP>ROCAUCPA (0.854 vs. $0.784, \mathrm{P}<0.05$, Table V; Fig. 1).

\section{Discussion}

As a complex clinical syndrome in the severe stage of various cardiovascular diseases, CHF has a very high incidence rate in the elderly, and is characterized by recurrent attack, continuous 
Table I. Comparison of baseline characteristics between MACE and non-MACE group.

\begin{tabular}{|c|c|c|c|c|}
\hline Variables & $\begin{array}{l}\text { MACE group } \\
\quad(n=249)\end{array}$ & $\begin{array}{l}\text { Non-MACE group } \\
\qquad(\mathrm{n}=161)\end{array}$ & $t / \chi^{2} / z$ & P-value \\
\hline Male, n (\%) & $118(47.39)$ & $75(46.58)$ & $\chi^{2}=1.35$ & 0.764 \\
\hline Age (years) & $77.82 \pm 6.57$ & $76.39 \pm 6.18$ & $\mathrm{t}=-3.36$ & 0.025 \\
\hline $\mathrm{HR}$ at admission (bpm) & $87.24 \pm 21.63$ & $82.76 \pm 16.52$ & $\mathrm{t}=-1.94$ & 0.043 \\
\hline NYHA stage (II \& III/IV, \%) & 93.02 & 71.28 & $\chi^{2}=5.27$ & 0.008 \\
\hline LVEDD (mm) & $50.54 \pm 14.62$ & $45.78 \pm 12.39$ & $\mathrm{t}=-2.58$ & 0.021 \\
\hline $\operatorname{LVEF}(\%)$ & $48.15 \pm 11.96$ & $52.97 \pm 11.06$ & $\mathrm{t}=4.92$ & 0.001 \\
\hline NT-pro BNP (pg/ml) & $8,864.52 \pm 9,718.36$ & $4,165.62 \pm 6,437.28$ & $z=-3.71$ & $<0.001$ \\
\hline cTNI & $1.56 \pm 5.42$ & $1.39 \pm 6.02$ & $t=-0.04$ & 0.892 \\
\hline ALB (g/l) & $35.29 \pm 4.14$ & $37.83 \pm 3.39$ & $\mathrm{t}=4.29$ & $<0.001$ \\
\hline PA (g/l) & $0.14 \pm 0.07$ & $0.19 \pm 0.05$ & $\mathrm{t}=5.76$ & $<0.001$ \\
\hline $\mathrm{Hb}(\mathrm{g} / \mathrm{l})$ & $113.27 \pm 23.54$ & $119.86 \pm 20.76$ & $\mathrm{t}=3.43$ & 0.019 \\
\hline $\mathrm{D}-\mathrm{D}(\mu \mathrm{g} / \mathrm{l})$ & $638.93 \pm 931.12$ & $544.26 \pm 749.83$ & $\mathrm{t}=-2.15$ & 0.341 \\
\hline Hct $(\%)$ & $34.16 \pm 6.39$ & $36.57 \pm 6.62$ & $\mathrm{t}=4.94$ & 0.037 \\
\hline $\mathrm{TC}(\mathrm{mmol} / \mathrm{l})$ & $3.82 \pm 1.07$ & $4.33 \pm 1.14$ & $\mathrm{t}=5.13$ & 0.002 \\
\hline TG (mmol/l) & $1.09 \pm 0.53$ & $1.48 \pm 0.87$ & $\mathrm{t}=6.02$ & $<0.001$ \\
\hline TBIL $(\mu \mathrm{mol} / \mathrm{l})$ & $16.46 \pm 9.82$ & $13.05 \pm 7.18$ & $\mathrm{t}=-3.58$ & 0.035 \\
\hline ALT (U/l) & $56.49 \pm 98.25$ & $39.62 \pm 70.54$ & $\mathrm{t}=-0.38$ & 0.467 \\
\hline AST (U/l) & $61.54 \pm 125.39$ & $54.63 \pm 160.36$ & $\mathrm{t}=-0.54$ & 0.926 \\
\hline $\operatorname{Scr}(\mu \mathrm{mol} / 1)$ & $104.64 \pm 65.37$ & $95.35 \pm 57.42$ & $\mathrm{t}=-2.76$ & 0.046 \\
\hline $\mathrm{UA}(\mu \mathrm{mol} / 1)$ & $417.81 \pm 169.26$ & $398.05 \pm 150.48$ & $\mathrm{t}=-1.33$ & 0.317 \\
\hline Cys C (mg/l) & $1.64 \pm 0.78$ & $1.43 \pm 0.62$ & $\mathrm{t}=-2.15$ & 0.036 \\
\hline hs-CRP (ng/ml) & $20.63 \pm 85.12$ & $15.32 \pm 60.81$ & $\mathrm{t}=-1.17$ & 0.418 \\
\hline Hospital stay (days) & $12.68 \pm 5.34$ & $11.26 \pm 3.72$ & $t=-1.92$ & 0.072 \\
\hline
\end{tabular}

Table II. Univariate regression analysis of prognosis in patients with $\mathrm{CHF}$.

\begin{tabular}{|c|c|c|c|}
\hline Variables & OR & $95 \% \mathrm{CI}$ & P-value \\
\hline NYHA (\%, II \& III/IV) & 1.926 & $1.174-2.985$ & 0.005 \\
\hline $\operatorname{LVEDD}(\mathrm{mm},>48.23$ vs. $\leq 48.23)$ & 1.847 & $1.214-2.892$ & 0.017 \\
\hline LVEF (\%, >51.69 vs. $\leq 51.69)$ & 0.623 & $0.357-0.931$ & 0.024 \\
\hline NT-pro BNP (pg/ml >3,305.46 vs. $\leq 3,305.46)$ & 3.059 & $1.758-5.027$ & $<0.001$ \\
\hline $\operatorname{ALB}(\mathrm{g} / \mathrm{l},>36.50$ vs. $\leq 36.50)$ & 0.417 & $0.275-0.618$ & $<0.001$ \\
\hline $\mathrm{PA}(\mathrm{g} / \mathrm{l}>0.16$ vs. $\leq 0.16)$ & 0.224 & $0.135-0.349$ & $<0.001$ \\
\hline $\mathrm{Hb}(\mathrm{g} / \mathrm{l}>118$ vs. $\leq 118)$ & 0.502 & $0.259-0.649$ & 0.009 \\
\hline $\operatorname{Hct}(\%,>36.32$ vs. $\leq 36.32)$ & 0.475 & $0.312-0.796$ & 0.007 \\
\hline $\mathrm{TC}(\mathrm{mmol} / 1,>3.83$ vs. $\leq 3.83)$ & 0.538 & $0.346-0.822$ & 0.004 \\
\hline
\end{tabular}

$\mathrm{P}<0.05$ indicates statistical significance. NYHA, New York heart association; LVEDD, left ventricular diastolic dimension; LVEF, left ventricular ejection fraction; NT-pro BNP, N-terminal pro-B-type natriuretic peptide; ALB, albumin; PA, pre-albumin; Hb, hemoglobin; Hct, hematocrit; TC, total cholesterol.

aggravation, multiple hospitalizations and high mortality rate (2). Therefore, early detection and treatment of $\mathrm{CHF}$ in the elderly patients is of great significance for improving the survival rate of the patients. In the present study, MACE occurred in 249 cases during follow-up, accounting for $60.73 \%$ of the total subjects, and there were 88 cases of all-cause death, 
Table III. Multivariate regression analysis of prognosis in patients with CHF.

\begin{tabular}{lccc}
\hline Variables & OR & $95 \%$ CI & P-value \\
\hline NYHA $(\%$, II \& III/ IV) & 1.952 & $1.471-3.383$ & 0.420 \\
LVEDD (mm, $>48.23$ vs. $\leq 48.23)$ & 1.548 & $1.062-2.891$ & 0.062 \\
LVEF $(\%,>51.69$ vs. $\leq 51.69)$ & 0.617 & $0.324-1.225$ & 0.323 \\
NT-pro BNP (pg/ml, $>3,305.46$ vs. $\leq 3,305.46)$ & 1.847 & $1.024-3.158$ & 0.036 \\
ALB $(\mathrm{g} / \mathrm{l},>36.50$ vs. $\leq 36.50)$ & 0.415 & $0.246-1.029$ & 0.052 \\
PA $(\mathrm{g} / \mathrm{l},>0.16$ vs. $\leq 0.16)$ & 0.242 & $0.137-0.406$ & $<0.001$ \\
Hb $(\mathrm{g} / \mathrm{l},>118$ vs. $\leq 118)$ & 0.416 & $0.198-1.132$ & 0.085 \\
Hct $(\%,>36.32$ vs. $\leq 36.32)$ & 0.625 & $0.423-1.819$ & 0.327 \\
TC $(\mathrm{mmol} / 1,>3.83$ vs. $\leq 3.83)$ & 0.834 & $0.697-1.238$ & 0.131 \\
\hline
\end{tabular}

$\mathrm{P}<0.05$ indicates statistical significance. NYHA, New York heart association; LVEDD, left ventricular diastolic dimension; LVEF, left ventricular ejection fraction; NT-pro BNP, N-terminal pro-B-type natriuretic peptide; ALB, albumin; PA, pre-albumin; Hb, hemoglobin; Hct, hematocrit; TC, total cholesterol.

Table IV. Comparison of heart failure severity and prognosis between PA $\leq 0.16 \mathrm{~g} / \mathrm{l}$ and PA $>0.16 \mathrm{~g} / \mathrm{l} \mathrm{CHF}$ patients.

\begin{tabular}{lccr}
\hline Variables & $\mathrm{PA} \leq 0.16 \mathrm{~g} / \mathrm{l}(\mathrm{n}=198)$ & $\mathrm{PA}>0.16 \mathrm{~g} / \mathrm{l}(\mathrm{n}=212)$ & $\mathrm{t} / \chi^{2} / \mathrm{z}$ \\
\hline Age (years) & $78.74 \pm 6.73$ & $76.21 \pm 6.16$ & $\mathrm{t}=-3.47$ \\
NYHA stage (II \& III/IV, \%) & 94.53 & 140.30 & 0.035 \\
MACE (\%) & 79.14 & 37.61 & 0.023 \\
NT-pro BNP (pg/ml) & $9287.46 \pm 10164.82$ & $4,895.37 \pm 6,910.58$ & $\chi^{2}=4.65$ \\
\end{tabular}

$\mathrm{P}<0.05$ indicates statistical significance. NYHA, New York heart association; MACE, major adverse cardiac events; NT-pro BNP, N-terminal pro-B-type natriuretic peptide; PA, pre-albumin.

Table V. Receiver operating characteristic (ROC) curve of PA and NT-pro BNP in predicting MACE events in patients with CHF.

\begin{tabular}{|c|c|c|c|c|c|}
\hline Variables & AUC & SE & $95 \% \mathrm{CI}$ & Sensitivity & Specificity \\
\hline NT-pro BNP (pg/ml) & 0.784 & 0.056 & $0.695-0.863$ & $77.65 \%$ & $69.12 \%$ \\
\hline $\mathrm{PA}(\mathrm{g} / \mathrm{l})$ & 0.845 & 0.031 & $0.726-0.938$ & $84.32 \%$ & $76.57 \%$ \\
\hline
\end{tabular}

NT-pro BNP, N-terminal pro-B-type natriuretic peptide; PA, pre-albumin.

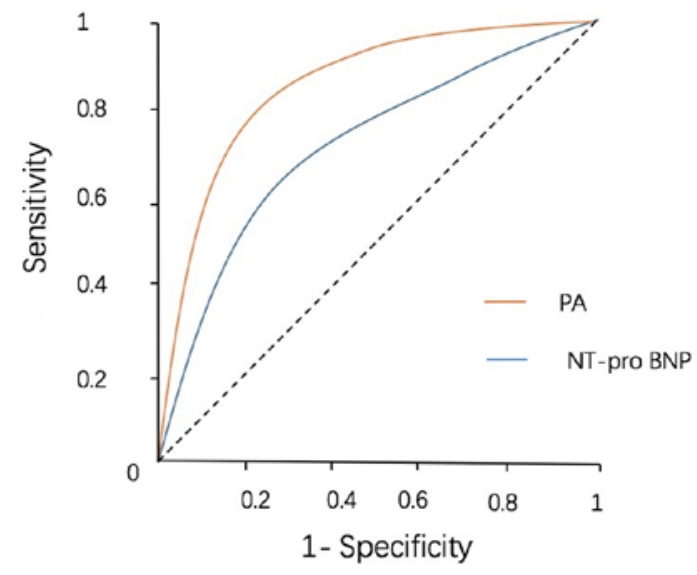

Figure 1. Receiver operating characteristic (ROC) curve of PA and NT-pro BNP in predicting MACE events in patients with CHF. PA, pre-albumin; NT-pro BNP, N-terminal pro-B-type natriuretic peptide. representing $21.46 \%$ of the total subjects. Comparisons were made between MACE group and non-MACE group, and it was found that the age, cardiac function grade based on the NYHA classification standards and NT-pro BNP were positively correlated with the occurrence of MACE, while ALB and PA levels were negatively associated with the occurrence of MACE.

NT-pro BNP is a non-bioactive polypeptide that is secreted by cardiomyocytes and enters the blood circulation through cell stretching. Under normal conditions, NT-pro BNP is mainly derived from atrial tissues, with a lower circulation level, while its level will be obviously raised under such pathophysiological conditions as altered ventricular volume, increased ventricular wall tension and myocardial ischemia and hypoxia. Previous studies have found that $\mathrm{CHF}$ patients with different grades of cardiac function of the 
NYHA standards exhibit an obvious inter-group difference in NT-pro BNP level, but NT-pro level varies dramatically among individuals and its measurement can be affected by multiple factors such as sex, age and body mass (7-10). For example, diuretic and vasoactive drugs produce great influences on the level of circulating NT-pro BNP. According to the results of this study, the level of NT-pro BNP was obviously higher in MACE group than that in non-MACE group, and the results of univariate and multivariate regression analysis revealed that the increase in NT-pro BNP level was significantly correlated with the occurrence of MACE in elderly CHF patients, implying that NT-pro BNP level is closely associated with the prognosis of heart failure, and that serum NT-pro BNP level can be monitored to effectively judge the prognosis of CHF. Although NT-pro BNP has a favorable application value in the severity grading and prognosis evaluation of $\mathrm{CHF}$, there is no set threshold of NT-pro BNP for predicting acute onset of CHF, which remains to be further explored.

Previous studies have demonstrated that malnutrition is an independent predictor for adverse cardiac events in $\mathrm{CHF}$ patients, and it is closely associated with CHF in the elderly as well. ALB is commonly used as the indicator for monitoring nutrition level, and a lower level of ALB has been found in some studies to serve as a risk factor for the poor prognosis of CHF patients, but with a longer half-life, ALB is not sensitive in reflecting short-term acute malnutrition (11). PA is an indicator for the early detection of malnutrition, and determining the concentration of plasma PA, more sensitive than ALB, has a special diagnostic value for understanding malnutrition, liver and kidney dysfunctions and disease severity (12-14). One study measured the level of serum PA in patients with renal failure, and the results manifested that it can be used as one of the highly sensitive indicators reflecting the nutrition status and survival rate of renal failure patients. Moreover, serum PA level declines in the patients with AMI and can be used for judging the conditions and prognosis of such patients, and it is also related to the short-term prognosis of patients with cerebral infarction (5). Besides, the study of Lourenço et al (6) revealed that the concentration of serum PA can promptly reflect the nutrition status in organisms, and it declines as cardiac function is weakened. Additionally, the 6 month mortality rate is obviously raised in HR patients with $\mathrm{PA}<150 \mathrm{mg} / \mathrm{l}$. It was found in the present study that $\mathrm{PA} \leq 0.16 \mathrm{~g} / \mathrm{l}$ group had higher plasma NT-pro BNP level and incidence rate of MACE, suggesting that low PA level is positively correlated with the severity of HR in the elderly, consistent with the results of the above-mentioned studies. The results of the study of Cabassi et al (15) manifested that PA is more accurate in predicting the death risk of the elderly CHF patients with a higher level of BNP, and it can be used for recognizing the high-risk CHF patients who need nutritional support. In the present study, multivariate regression analysis was performed with the cardiac events as the dependent variable, and it was discovered that decline in PA level is strongly associated with the occurrence of MACE.

PA is more sensitive in evaluating early changes in nutrition level, and it can be used for predicting heart failure probably because worsened HF impairs liver function and raises the secretion of inflammatory factors through the chronic inflammatory process, thereby increasing consumption of PA and decreasing its synthesis, and ultimately reducing PA level. Therefore, PA level is valuable in predicting the prognosis to a certain degree.

Blood PA and NT-pro BNP levels are effective indicators for predicting the occurrence of cardiac events in elderly CHF patients, and they are of great clinical significance for evaluating the severity and prognosis of the disease. However, lack of ECG data and time recording for MACE follow-up is a limitation of present study, time data should be supplemented in the future research to permit time-based analysis and Cox regression. Since CHF is a complex clinical syndrome due to the synergy of multiple factors, and the present study has a relative small sample size, the specific mechanisms of action of changes in blood PA and NT-pro BNP levels in elderly CHF patients remain to be further explored.

In conclusion, the levels of PA and NT-proBNP are significantly related to the severity of heart failure in elderly CHF patients. The detection of PA and NT-proBNP has guiding significance in evaluating the prognosis of elderly patients with CHF.

\section{Acknowledgements}

Not applicable.

Funding

Not applicable.

\section{Availability of data and materials}

All data generated or analyzed during this study are included in this published article.

\section{Authors' contributions}

LS, MC and XY designed the study and performed the experiments. LS and YZ established the animal models. MC and JZ collected the data. YG and JL analyzed the data. LS, MC and $\mathrm{XY}$ prepared the manuscript. All authors read and approved the final manuscript.

\section{Ethics approval and consent to participate}

The current study was approved by the Ethics Committee of Beijing Chaoyang Hospital (Beijing, China). Patients who participated in this research had complete clinical data. The signed informed consents were obtained from the patients or the guardians.

\section{Patient consent for publication}

Not applicable.

\section{Competing interests}

The authors declare they have no competing interests. 


\section{References}

1. Schulz M, Griese-Mammen N, Anker SD, Koehler F, Ihle P, Ruckes C, Schumacher PM, Trenk D, Böhm M, Laufs U, et al; PHARM-CHF Investigators: Pharmacy-based interdisciplinary intervention for patients with chronic heart failure: Results of the PHARM-CHF randomized controlled trial. Eur J Heart Fail 21: 1012-1021, 2019.

2. Mancini A, Vergani E, Bruno C, Olivieri G, Di Segni C, Silvestrini A, Venuti A, Favuzzi A and Meucci E: Oxidative stress as a possible mechanism underlying multi-hormonal deficiency in chronic heart failure. Eur Rev Med Pharmacol Sci 22: 3936-3961, 2018.

3. He WM, Luo YT, Shui X, Liao XX, Liu JL and Zhuang XD: Critical appraisal of international guidelines on chronic heart failure: Can China AGREE? Int J Cardiol 203: 111-114, 2016.

4. Fan L, Chi C, Guo S, Wang Y, Cai W, Shao X, Xu F, Pan J, Zhu Y, Shangguan $X$, et al: Serum pre-albumin predicts the clinical outcome in metastatic castration-resistant prostate cancer patients treated with abiraterone. J Cancer 8: 3448-3455, 2017.

5. Cubedo J, Padró T, Alonso R, Cinca J, Mata P and Badimon L: Differential proteomic distribution of TTR (pre-albumin) forms in serum and HDL of patients with high cardiovascular risk. Atherosclerosis 222: 263-269, 2012.

6. Lourenço P, Silva S, Friões F, Alvelos M, Amorim M, TorresRamalho P, Teles MJ, Guimarães JT and Bettencourt P: Does pre-albumin predict in-hospital mortality in heart failure? Int J Cardiol 166: 758-760, 2013.

7. Whitman IR, Vittinghoff E, DeFilippi CR, Gottdiener JS, Alonso A, Psaty BM, Heckbert SR, Hoogeveen RC, Arking DE, Selvin E, et al: NT-pro-BNP as a mediator of the racial difference in incident atrial fibrillation and heart failure. J Am Heart Assoc 8: e010868, 2019.

8. Letsas KP, Filippatos GS, Pappas LK, Mihas CC, Markou V, Alexanian IP, Efremidis M, Sideris A, Maisel AS and Kardaras F: Determinants of plasma NT-pro-BNP levels in patients with atrial fibrillation and preserved left ventricular ejection fraction. Clin Res Cardiol 98: 101-106, 2009.

9. Olsson LG, Swedberg K, Cleland JG, Spark PA, Komajda M, Metra M, Torp-Pedersen C, Remme WJ, Scherhag A and PooleWilson P; COMET Investigators: Prognostic importance of plasma NT-pro-BNP in chronic heart failure in patients treated with a beta-blocker: Results from the Carvedilol Or Metoprolol European Trial (COMET) trial. Eur J Heart Fail 9: 795-801, 2007.
10. Pfister $R$, Tan D, Thekkanal J, Hellmich $M$ and Schneider CA: Predictors of elevated NT-pro-BNP in cardiovascular patients without acute heart failure. Int $\mathrm{J}$ Cardiol 131: 277-280, 2009.

11. Gotsman I, Shauer A, Zwas DR, Tahiroglu I, Lotan C and Keren A: Low serum albumin: A significant predictor of reduced survival in patients with chronic heart failure. Clin Cardiol 42: 365-372, 2019.

12. Player EL, Morris P, Thomas T, Chan WY, Vyas R, Dutton J, Tang J, Alexandre L and Forbes A: Bioelectrical impedance analysis (BIA)-derived phase angle (PA) is a practical aid to nutritional assessment in hospital in-patients. Clin Nutr 38: 1700-1706, 2019

13. Fattah SA, El-Hamshary NK, Kilany YF, Salem MN, Ismael SA, Mostafa BD and El-Dessouky YM: Prognostic and predictive values of MELD score, platelet count and pre-albumin in patients with compensated and decompensated liver cirrhosis with acute variceal bleeding. J Egypt Soc Parasitol 42: 443-452, 2012.

14. Ruperto M, Sánchez-Muniz FJ and Barril G: Predictors of protein-energy wasting in haemodialysis patients: A crosssectional study. J Hum Nutr Diet 29: 38-47, 2016.

15. Cabassi A, de Champlain J, Maggiore U, Parenti E, Coghi P, Vicini V, Tedeschi S, Cremaschi E, Binno S, Rocco R, et al: Prealbumin improves death risk prediction of BNP - added Seattle Heart Failure Model: Results from a pilot study in elderly chronic heart failure patients. Int J Cardiol 168: 33343339, 2013.

(i) $($ This work is licensed under a Creative Commons Attribution-NonCommercial-NoDerivatives 4.0 International (CC BY-NC-ND 4.0) License. 